\title{
Caractéristiques physico-chimiques des eaux de forages à usage domestique dans la ville de Daloa (centre-ouest de la Côte d'Ivoire)
}

\author{
Yao Francis KOUAME ${ }^{1 *}$, Atolé Brice Bienvenu KEDI ${ }^{1}$, Seka Simplice KOUASSI ${ }^{1}$, \\ N'Guessan Jimmy Aristide KONAN ${ }^{1}$, Egomli Stanislas ASSOHOUN ${ }^{1}$, \\ Ossey Bernard $\mathrm{YAPO}^{2}$ et Théophile GNAGNE ${ }^{3}$
}

\author{
${ }^{1}$ Laboratoire des Sciences et Technologie de l'Environnement, UFR Environnement, Université Jean \\ Lorougnon Guédé de Daloa BP 150 Daloa, Côte d'Ivoire. \\ ${ }^{2}$ Laboratoire des Sciences de l'Environnement, UFR des Sciences et Gestion de l'Environnement, Université \\ Nangui Abrogoua 02 BP 801 Abidjan 02, Côte d'Ivoire. \\ ${ }^{3}$ Laboratoire Géosciences et Environnement, UFR des Sciences et Gestion de l'Environnement, Université \\ Nangui Abrogoua 02 B.P.801 Abidjan 02, Côte d'Ivoire. \\ *Auteur correspondant ; E-mail : franciskouameyao@yahoo.fr, Tél. : 002250757338911.
}

\begin{tabular}{lll}
\hline Received: 04-11-2020 & Accepted: 15-04-2021 & Published: 30-04-2021 \\
\hline
\end{tabular}

\section{RESUME}

Les populations de la ville de Daloa en Côte d'Ivoire consomment majoritairement les eaux de forages au détriment de l'eau fournie par la société agréée. Ainsi, cette étude des eaux de forages a pour objectif l'évaluation de ses caractéristiques physico-chimiques. Elle a permis de mesurer au niveau de quinze forages les paramètres tels que le $\mathrm{pH}$, la conductivité électrique, la température, le nitrite, le nitrate, l'ammonium, le sulfate, le bicarbonate, le calcium, le magnésium et le potassium. Les valeurs moyennes sont comparées aux normes relatives à la qualité de l'eau de boisson. L'analyse statistique multivariée dont l'Analyse en Composantes Principales (ACP) et la Classification Hiérarchique Ascendante (CHA) a été également appliquée à l'ensemble des paramètres mesurés. Il ressort des résultats que l'eau des forages est légèrement acide avec un $\mathrm{pH}$ moyen de $6,0 \pm 0,5$. Elle est faiblement minéralisée avec une conductivité électrique moyenne de 246,2 $\pm 162,6 \mu \mathrm{S} / \mathrm{cm}$. Une forte corrélation est signalée entre la conductivité électrique et les paramètres suivants : nitrate, ammonium, bicarbonate, calcium, magnésium, sodium et potassium. La classification des forages est gouvernée par les valeurs de conductivité et de $\mathrm{pH}$ qui permettent de regrouper les forages selon leur qualité physico-chimique. Les eaux des forages de Daloa sont conformes aux directives de l'OMS.

(C) 2021 International Formulae Group. All rights reserved.

Mots clés : Daloa, eau de forage, norme, paramètres physico-chimique, qualité de l'eau.

\section{Physicochemical characteristic of ground water for domestic use in the town of Daloa (Midwest, Ivory Coast)}

\begin{abstract}
The population of Daloa (third largest city in Côte d'Ivoire) mainly consume borehole water to the detriment of water provided by approved company. Thus, the quality of borehole water is evaluated from their physicochemical characteristics in this study. The study carried out in various districts of the city made it possible to measure for fifteen boreholes the physicochemical parameters such as $\mathrm{pH}$, electric conductivity, temperature,
\end{abstract}


nitrite, nitrate, ammonium, sulphate, bicarbonate, calcium, magnesium and potassium. The average values are compared with the standards relating to drinking water quality. The Multivariate statistical analysis whose Principal Components Analysis (PCA) and hierarchical clustering (HC) were also applied to the whole of the measured parameters. The results show that the borehole water is slightly acid with an average pH of $6,0 \pm 0,5$. It is slightly mineral-bearing with an average electric conductivity of $246,2 \pm 162,6 \mu \mathrm{S} / \mathrm{cm}$. A strong correlation is announced between electric conductivity and the following parameters: nitrate, ammonium, bicarbonate, calcium, magnesium, sodium and potassium. The classification of borehole controlled by this value of conductivity and $\mathrm{pH}$ which makes it possible to gather borehole according to their physicochemical quality. The physicochemical parameters of borehole water from Daloa are in conformity with the directives of WHO. (C) 2021 International Formulae Group. All rights reserved.

Keywords: Daloa, borehole water, standard, physicochemical parameters, water quality.

\section{INTRODUCTION}

Avoir accès à l'eau potable et à l'assainissement est un droit pour l'homme et un Objectif de Développement Durable (ONU, 2019). A ce propos, l'Organisation Mondiale de la Santé souligne que 1,1 milliards de personnes soit $17 \%$ de la population mondiale n'ont pas accès à l'eau potable et 2,6 milliards soit $42 \%$ n'ont pas accès à un assainissement adéquat (OMS, 2006). Aussi, selon les Nations Unies, environ deux millions de personnes, pour la plupart des enfants, meurent-ils chaque année des suites de maladie causées par une eau impropre à la consommation (ONU, 2019).

En Côte d'Ivoire, l'Etat a très tôt compris cette exigence et a développé depuis son indépendance une stratégie d'alimentation en eau potable qui a enregistré des résultats satisfaisants au fil des ans. Cette politique ponctuée par des réformes innovantes a permis selon les contextes, de structurer les besoins en trois sous-secteurs: l'hydraulique urbaine (HU), l'hydraulique villageoise (HV) et l'hydraulique villageoise améliorée (HVA).En plus, l'Etat dispose d'une Société de Distribution d'Eau potable (SODECI) qui est une pièce maitresse stratégique dans l'approvisionnement en eau, sans oublier l'Office National de l'Eau Potable (ONEP) et la Direction Générale de l'Approvisionnement en Eau potable (DGAE) pour leur rôle de gestionnaire du patrimoine, de développement des infrastructures hydrauliques et de régulateur du secteur de l'eau en Côte d'Ivoire. Nonobstant tous les efforts consentis par les autorités étatiques, les services d'eau potable enregistrent un taux de couverture nationale de
$48 \%$ en milieu rural et $70 \%$ en milieu urbain. Des efforts doivent être faits en vue d'accroître ce taux (Koné et al., 2018).

Daloa, ville du centre-ouest de Côte d'Ivoire connait un problème d'accès à l'eau potable (Aké et al., 2018). En effet les populations de la ville estiment que l'eau fournie par la SODECI n'est pas de bonne qualité. Ce constat est dû au goût peu agréable, à la couleur de l'eau et surtout au coût élevé du branchement et de l'abonnement au réseau de distribution. Par conséquent ces populations en plus de celles qui n'ont pas accès au réseau de la SODECI se tournent alors vers les eaux de forage. Si l'eau provenant des forages est en générale de bonne qualité (CICR, 2012), celle provenant de la ville de Daloa est-elle conforme aux directives de l'OMS (2011) en matière d'eau potable? L'objectif de ce travail est donc d'évaluer la qualité physico-chimique de l'eau de forages dans la ville de Daloa.

\section{MATERIEL ET METHODES \\ Zone d'étude}

Daloa est une ville située au centreouest de la Côte d'Ivoire dans la région du Haut Sassandra entre $6^{\circ} 53$ de latitude nord et $6^{\circ} 27$ de longitude ouest (Figure 1). Elle se trouve à $141 \mathrm{~km}$ de Yamoussoukro, la capitale politique et à $383 \mathrm{~km}$ d'Abidjan, la capitale économique. C'est la troisième ville la plus peuplée du pays après celle d'Abidjan et Bouaké. Elle a une population estimée à 266000 habitants (RGPH, 2014). Les principaux quartiers de la ville sont Abattoir 1 \& 2, Lobia 1 \& 2, Labia Gbeuliville, Tazibouo, Commerce, Marais, Orly, Kennedy, Soleil, Huberson, Manioc, 
Garage. Le climat est de type équatorial avec deux saisons pluvieuses et deux saisons sèches qui s'alternent. Les précipitations atteignent 1200 à $1400 \mathrm{~mm}$ par an. Daloa dispose à priori d'un potentiel pluviométrique pour la production d'eau potable nécessaire à population. Toutefois, ce potentiel subit des baisses depuis quelques années (Koukougnon, 2012). Le substrat géologique de la région du Haut Sassandra dont fait partie la ville de Daloa est granitique. Ce qui a donné naissance à des sols ferrugineux par endroit et à des sols de type ferralitique de texture argilo-sableux ou argileuse et riche en humus. Les sols sont favorables aux cultures pérennes, telles que les cultures du binôme café-cacao, la culture de l'hévéa et la culture du palmier à huile (Die, 2006). La ville est située dans le grand bassin du fleuve Sassandra avec pour affluent la rivière Lobo. Cette rivière est utilisée pour l'approvisionnement en eau potable de la ville (Koukougnon, 2012).

\section{Echantillonnage et analyse de l'eau}

L'échantillonnage a couvert différents quartiers de la ville. Les eaux de 15 forages sont prélevées selon le Tableau 1. Les paramètres physiques tels que la température, le $\mathrm{pH}$, et la conductivité ont été mesuré in situ à chaque point d'eau échantillonnée. Pour ces différents paramètres, les appareils de mesure préalablement calibrés sont mis sous tension quelques minutes avant toute manipulation. Les sondes sont plongées ensuite dans l'échantillon d'eau prélevé et la sélection de la fonction du paramètre désiré donne la valeur de ce dernier sur l'écran d'affichage. Les échantillons d'eau sont collectés dans des bouteilles en polyéthylène de 1litre. Chaque flacon est rincé et rempli avec l'eau prélevée. Tous ces flacons sont conservés dans une glacière et acheminés au laboratoire, où ils sont stockés à une température de $-5{ }^{\circ} \mathrm{C}$. Les méthodes d'analyses sont consignées dans le Tableau 2.

L'Analyse en Composantes Principales a été réalisée sur les résultats obtenus des paramètres physico-chimiques caractérisant les quinze forages (Tableau 1) à l'aide du logiciel libre $\mathrm{R}$ version 4.0.2. Cette analyse a permis de faire ressortir les corrélations existantes entre les différents paramètres de l'eau. Afin d'obtenir une meilleure affectation des individus à leur classe, cette analyse a été complétée par une Classification Hiérarchique Ascendante telle que préconisée par Belkhiri et al. (2010) toujours à l'aide du logiciel $\mathrm{R}$ version 4.0.2.

Tableau 1 : Répartition des forages selon les quartiers de la ville de Daloa.

\begin{tabular}{lcl}
\hline Quartiers & Nombre de forage & Identification des forages \\
\hline Abattoir 2 & 2 & F14; F15 \\
\hline Garage & 4 & F9; F10; F11; F12 \\
\hline Marais & 1 & F 8 \\
\hline Manioc mosquée & 1 & F13 \\
\hline Commerce & 1 & F4 \\
\hline CHR & 1 & F5 \\
\hline Lobia SODECI & 1 & F2 \\
\hline Lobia 2 & 1 & F3 \\
\hline Orly école centre trois & 1 & F7 \\
\hline Huberson rue ado & 1 & F6 \\
\hline Tazibouo institut pastorale & 1 & F1 \\
\hline
\end{tabular}



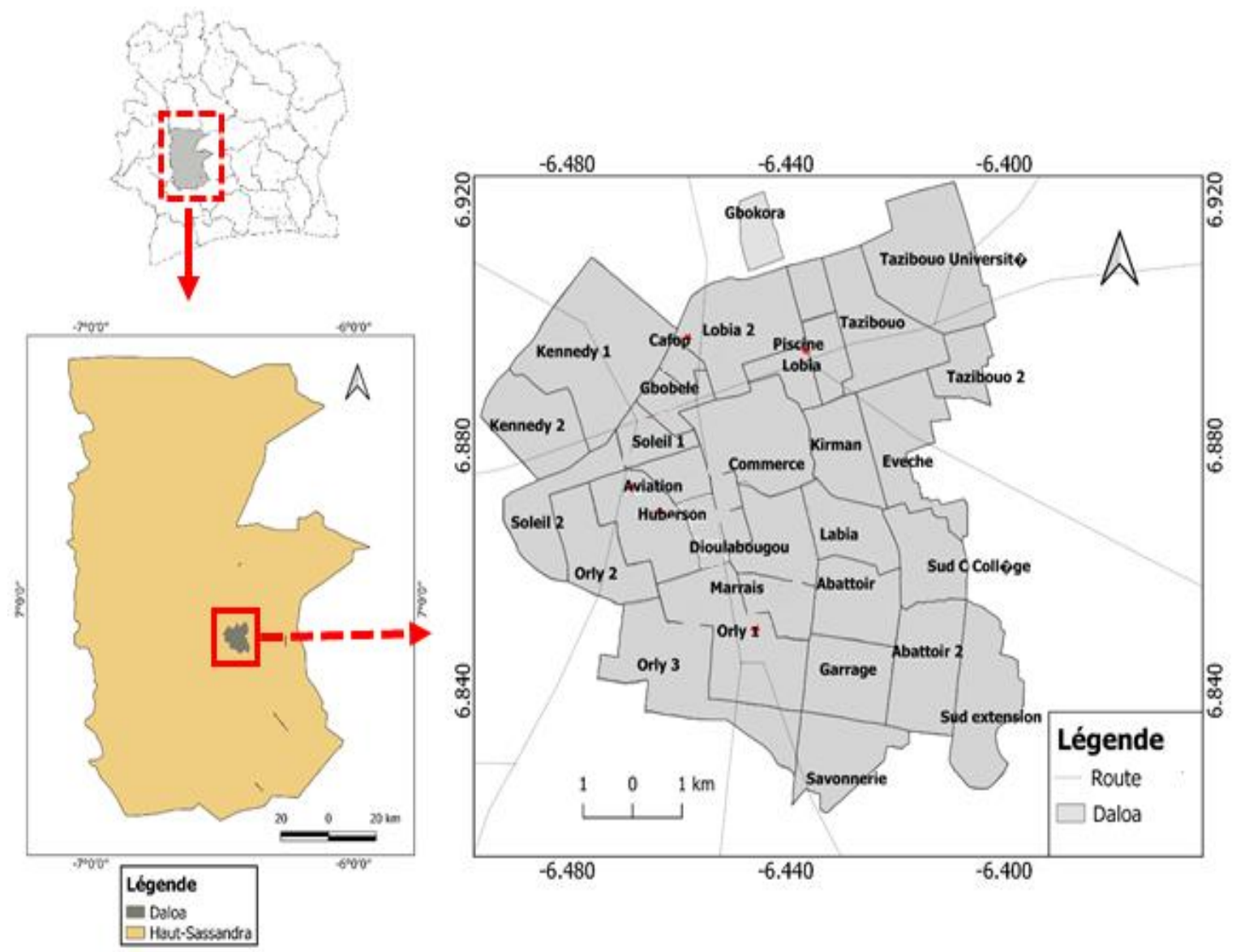

Figure 1 : Carte géographique de la ville de Daloa.

Tableau 2 : Méthodes d'analyses.

\begin{tabular}{ll}
\hline \multicolumn{1}{c}{ Paramètres } & \multicolumn{1}{c}{ Méthodes d'analyses } \\
\hline $\begin{array}{l}\text { pH ; Température } ; \\
\text { Conductivité }\end{array}$ & Electrochimique à l'électrode de verre (NF T 90-008) \\
\hline Ammonium & Spectrophotométrie au bleu d'indophénol (NF T 90-015) \\
\hline Nitrate & Spectrophotométrie au salicylate de sodium (NF T 90-045) \\
\hline Nitrite & $\begin{array}{l}\text { Spectrophotométrie d'absorption moléculaire de l'amino-4-benzène } \\
\text { sulfonamide en milieu acide (NF T 90-013) }\end{array}$ \\
\hline Sulfate & Chromatographie ionique (CEAEQ,2011) \\
\hline Sodium, Calcium, & $\begin{array}{l}\text { Spectrophotométrie de masse à source ionisante à plasma d'argon } \\
\text { Magnesium, Potassium }\end{array}$ \\
\hline
\end{tabular}




\section{RESULTATS}

\section{Caractéristiques physico-chimiques de l'eau}

Les résultats des analyses physicochimiques de l'eau des forages étudiés sont consignés dans le Tableau 3. Le Tableau 4 regroupe les normes de qualité de l'eau de boisson, les valeurs moyennes et extrêmes des différents paramètres physico-chimiques mesurées de l'eau des forages. La température de l'eau des forages de Daloa est en moyenne de $30,0 \pm 1,6^{\circ} \mathrm{C}$. Elle est comprise entre 27,5 ${ }^{\circ} \mathrm{C}$ et $33,5{ }^{\circ} \mathrm{C}$. La conductivité électrique a donné des valeurs comprises entre $86,5 \mu \mathrm{S} / \mathrm{cm}$ et $555 \mu \mathrm{S} / \mathrm{cm}$ avec une moyenne de $246,2 \pm$ $162,6 \mu \mathrm{S} / \mathrm{cm}$ pour l'ensemble des forages étudiés. Le pH est compris entre 5,0 et 6,9 avec pour valeur moyenne $6,0 \pm 0,5$. Les concentrations en nitrite sont très faibles. La valeur la plus élevée est de $0,01 \mathrm{mg} / \mathrm{L}$. Les nitrates varient de $6,1 \mathrm{mg} / \mathrm{L}$ à $114,3 \mathrm{mg} / \mathrm{L}$ avec une moyenne de $41,6 \pm 38,9 \mathrm{mg} / \mathrm{L}$. Les concentrations en ammonium varient de 0,1 $\mathrm{mg} / \mathrm{L}$ à $2,6 \mathrm{mg} / \mathrm{L}$ avec une moyenne de $0,5 \pm$ $0,8 \mathrm{mg} / \mathrm{L}$. L'azote dans ces eaux de forage est conforme à la valeur maximale admissible recommandée par l'Organisation Mondiale de la Santé (2011). Les composés suivants présentent des moyennes suivantes : le sulfate $(33,7 \pm 82,5 \mathrm{mg} / \mathrm{L})$, le calcium $(5,9 \pm 6,7$ $\mathrm{mg} / \mathrm{L})$, le magnésium $(2,3 \pm 1,5 \mathrm{mg} / \mathrm{L})$, le sodium $(11,7 \pm 6,7 \mathrm{mg} / \mathrm{L})$ et le potassium $(6,3$ $\pm 8,5 \mathrm{mg} / \mathrm{L})$. Comme l'azote, ces composés présentent des valeurs en dessous des normes de l'OMS. Les paramètres physico-chimiques étudiés des eaux des forages de la ville de Daloa sont conformes aux directives de l'OMS.

\section{Corrélations entre les différents paramètres physico-chimiques de l'eau}

L'Analyse en Composantes Principales réalisée sur les 12 paramètres physicochimiques montre que les deux axes cumulent
$67,2 \%$ de la variance totale (le premier axe explique $39,22 \%$ et le deuxième axe $27,98 \%$ de cette variance). L'axe 1 est fortement corrélé du côté positif avec la conductivité, l'ammonium, le bicarbonate, le sodium et le potassium. A l'inverse, l'axe 2 est fortement corrélé du côté négatif avec le nitrite et le nitrate et corrélé du côté positif avec le $\mathrm{pH}$, le sulfate et le calcium (Figure 2).

L'examen de la matrice de corrélation entre variables (Tableau 5) révèle la présence de variables biens corrélées entre elles. Il s'agit de :

$\checkmark$ Conductivité avec les paramètres, nitrate, ammonium, bicarbonate, calcium, magnésium, sodium et potassium ;

$\checkmark$ pH avec le nitrate, le calcium et le magnésium ;

$\checkmark$ Nitrate avec l'ammonium, le sodium et le potassium ;

$\checkmark$ Ammonium avec le bicarbonate, le sodium et le potassium ;

$\checkmark$ Sulfate avec le calcium;

$\checkmark$ Bicarbonate avec le potassium;

$\checkmark$ Calcium avec le magnésium ;

$\checkmark$ Sodium avec le potassium.

Le dendrogramme représentant la classification hiérarchique des forages obtenue à partir des données physico-chimiques fait apparaitre 3 classes. La classe 1 est composée des forages 4 et 7 . Elle est caractérisée par les plus fortes valeurs de conductivité, de concentration, nitrate, ammonium, bicarbonate, sodium et potassium. La classe 2 est composée des forages 5, 9, 10 et 11 . Elle est caractérisée par les plus fortes valeurs de $\mathrm{pH}$, de concentration, de calcium et de magnésium. La classe 3 est composée des forages 1, 2, 3, 6, $8,12,13,14$ et 15 . Elle est caractérisée par les faibles valeurs de concentration de $\mathrm{pH}$, conductivité, calcium, magnésium et bicarbonate. 
Tableau 3 : Valeurs des paramètres physico-chimiques des eaux de forages de la ville de Daloa.

\begin{tabular}{|c|c|c|c|c|c|c|c|c|c|c|c|c|}
\hline $\mathbf{N}^{\circ}$ Echantillons & $\mathbf{p H}$ & $\begin{array}{c}\text { Temp } \\
\left({ }^{\circ} \mathbf{C}\right)\end{array}$ & $\begin{array}{l}\text { Cond } \\
(\mu \mathrm{S} / \mathrm{cm})\end{array}$ & $\begin{array}{c}\mathrm{NO}_{2}^{-} \\
(\mathrm{mg} / \mathrm{L})\end{array}$ & $\begin{array}{c}\mathrm{NO}_{3}^{-} \\
(\mathrm{mg} / \mathrm{L})\end{array}$ & $\begin{array}{c}\mathrm{NH}_{4}^{+} \\
(\mathrm{mg} / \mathrm{L})\end{array}$ & $\begin{array}{l}\mathrm{SO}_{4}{ }^{2-} \\
(\mathrm{mg} / \mathrm{L})\end{array}$ & $\begin{array}{c}\mathrm{HCO}_{3}^{-} \\
(\mathrm{mg} / \mathrm{L})\end{array}$ & $\begin{array}{c}\mathrm{Ca}^{2+} \\
(\mathrm{mg} / \mathrm{L})\end{array}$ & $\begin{array}{c}\mathrm{Mg}^{2+} \\
(\mathrm{mg} / \mathrm{L})\end{array}$ & $\begin{array}{c}\mathbf{N a}^{+} \\
(\mu \mathrm{g} / \mathrm{L})\end{array}$ & $\begin{array}{c}\mathbf{K}^{+} \\
(\mathbf{m g} / \mathbf{L})\end{array}$ \\
\hline F1 & 5,97 & 29,40 & 115,40 & 0,000 & 32,50 & 0,17 & 0,00 & 24,00 & 1,21 & 1,56 & 6,87 & 3,13 \\
\hline $\mathrm{F} 2$ & 5,42 & 27,50 & 261,00 & 0,002 & 112,70 & 0,14 & 2,87 & 25,00 & 3,91 & 1,26 & 15,24 & 6,39 \\
\hline F3 & 4,96 & 30,70 & 92,60 & 0,006 & 42,70 & 0,24 & 0,00 & 28,00 & $<0,5$ & $<0,5$ & 7,04 & 1,65 \\
\hline $\mathrm{F} 4$ & 5,29 & 29,00 & 555,00 & 0,000 & 114,30 & 2,54 & 8,69 & 78,00 & 9,80 & 3,78 & 31,40 & 35,90 \\
\hline F5 & 6,91 & 28,90 & 311,70 & 0,004 & 11,40 & 0,13 & 0,77 & 45,00 & 11,70 & 4,33 & 16,00 & 2,56 \\
\hline F6 & 6,17 & 33,00 & 100,80 & 0,000 & 6,10 & 0,18 & 0,00 & 42,00 & 1,94 & 1,60 & 4,35 & 2,75 \\
\hline F7 & 6,25 & 31,00 & 513,13 & 0,007 & 110,90 & 2,59 & 4,06 & 85,00 & 2,31 & 1,65 & 18,04 & 11,90 \\
\hline F8 & 5,93 & 30,50 & 214,10 & 0,000 & 44,50 & 0,16 & 0,00 & 30,00 & 4,20 & 3,77 & 12,00 & 4,82 \\
\hline F9 & 6,49 & 29,70 & 308,50 & 0,000 & 23,20 & 0,16 & 179,37 & 35,00 & 14,50 & 2,63 & 13,70 & 3,52 \\
\hline F10 & 6,75 & 30,00 & 493,50 & 0,002 & 14,70 & 0,18 & 281,71 & 76,00 & 24,30 & 4,01 & 9,62 & 3,93 \\
\hline F11 & 6,57 & 29,80 & 261,00 & 0,000 & 9,60 & 0,15 & 4,41 & 71,00 & 8,16 & 4,94 & 11,70 & 5,03 \\
\hline F12 & 5,73 & 33,50 & 167,50 & 0,009 & 46,42 & 0,15 & 0,00 & 55,00 & 2,03 & 1,83 & 8,2 & 4,32 \\
\hline F13 & 5,86 & 29,20 & 97,30 & 0,002 & 15,00 & 0,12 & 23,47 & 62,00 & 1,14 & 0,81 & 6,96 & 3,07 \\
\hline F14 & 5,93 & 28,70 & 115,40 & 0,010 & 24,70 & 0,13 & 0,00 & 45,00 & 1,27 & 0,94 & 8,55 & 3,45 \\
\hline F15 & 5,81 & 29,00 & 86,50 & 0,000 & 15,00 & 0,15 & 0,00 & 35,00 & 1,01 & 0,69 & 6,56 & 2,73 \\
\hline
\end{tabular}


Tableau 4 : Comparaison des moyennes des paramètres physico-chimiques avec les normes.

\begin{tabular}{lllllll}
\hline Paramètres & Unités & $\begin{array}{l}\text { Norme } \\
\text { OMS }\end{array}$ & Minimum & Maximum & Médiane & $\begin{array}{l}\text { Moyenne + } \\
\text { écart-type }\end{array}$ \\
\hline $\mathbf{p H}$ & & $6,5-8,5$ & 5 & 6,9 & 5,9 & $6 \pm 0,5$ \\
\hline Température & ${ }^{\circ} \mathrm{C}$ & $25-30$ & 27,5 & 33,5 & 29,7 & $30 \pm 1,6$ \\
\hline Conductivité & $\mu \mathrm{S} / \mathrm{cm}$ & $500-1500$ & 86,5 & 555 & 214,1 & 246,2 \\
\hline $\mathbf{N O 2}^{-}$ & $\mathrm{mg} / \mathrm{L}$ & 3 & 0,0 & 0,0 & 0,0 & 0,0 \\
\hline $\mathbf{N O}_{\mathbf{3}}{ }^{-}$ & $\mathrm{mg} / \mathrm{L}$ & 50 & 6,1 & 114,3 & 24,7 & $41,6 \pm 38,9$ \\
\hline $\mathbf{N H}_{4}{ }^{+}$ & $\mathrm{mg} / \mathrm{L}$ & 0,5 & 0,1 & 2,6 & 0,2 & $0,5 \pm 0,8$ \\
\hline $\mathbf{S O}_{4} \mathbf{2}^{-}$ & $\mathrm{mg} / \mathrm{L}$ & 250 & 0,0 & 281,7 & 0,8 & $33,7 \pm 82,5$ \\
\hline $\mathbf{H C O}_{\mathbf{3}}{ }^{-}$ & $\mathrm{mg} / \mathrm{L}$ & - & 24 & 85 & 45 & $49,1 \pm 20,8$ \\
\hline $\mathbf{C a 2 +}$ & $\mathrm{mg} / \mathrm{L}$ & 100 & 0,5 & 24,3 & 2,3 & $5,9 \pm 6,7$ \\
\hline $\mathbf{M g 2 +}$ & $\mathrm{mg} / \mathrm{L}$ & -150 & 0,5 & 4,9 & 1,6 & $2,3 \pm 1,5$ \\
\hline $\mathbf{N a}+$ & $\mu \mathrm{g} / \mathrm{L}$ & - & 4,3 & 31,4 & 9,6 & $11,7 \pm 6,7$ \\
\hline $\mathbf{K}+$ & $\mathrm{mg} / \mathrm{L}$ & -200 & 1,6 & 35,9 & 3,5 & $6,3 \pm 8,5$ \\
\hline
\end{tabular}

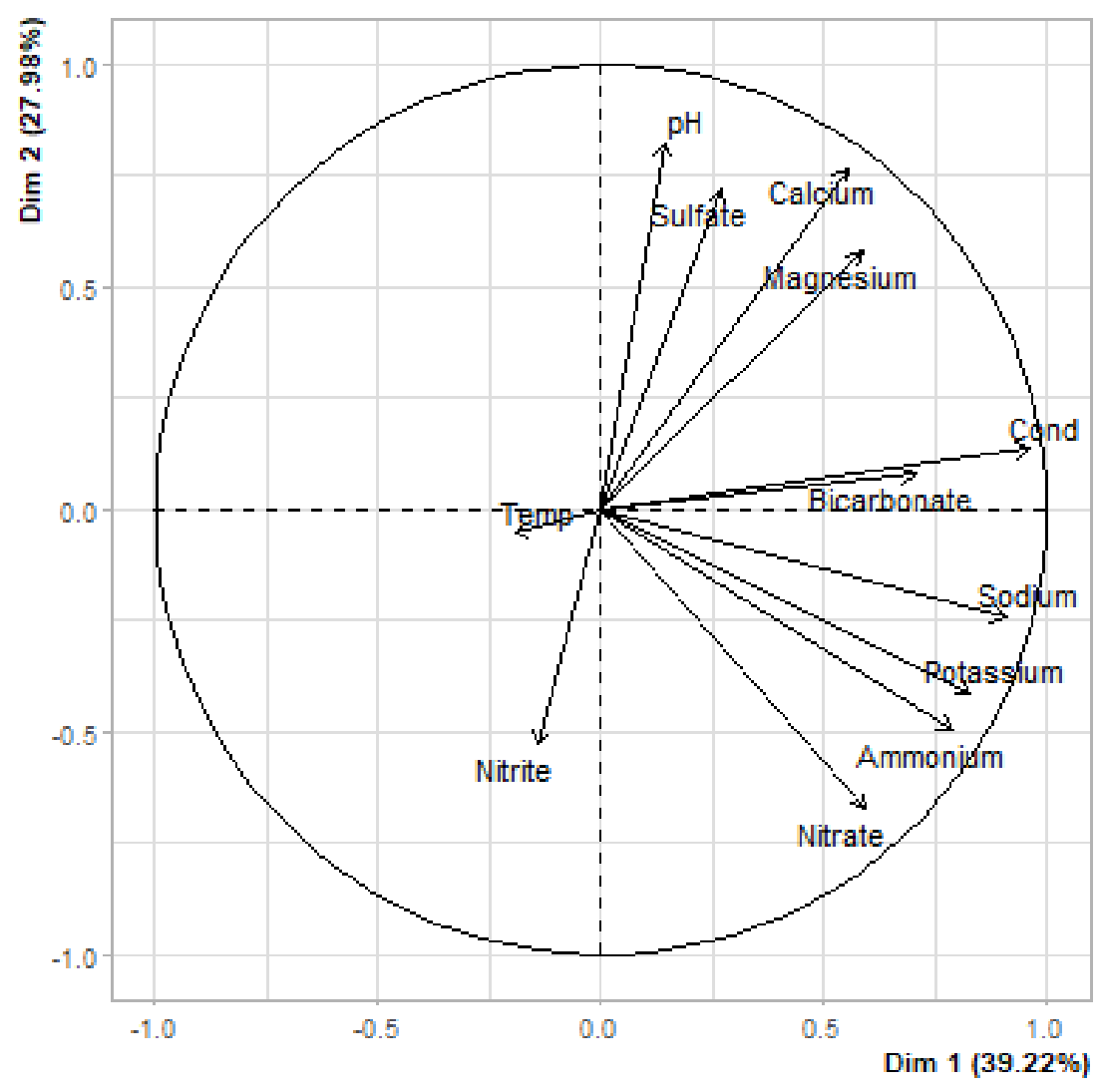

Figure 2 : Carte factorielle des variables. 
Tableau 5 : Matrice de corrélation entre les variables physico-chimiques sur l'ensemble des forages.

\begin{tabular}{|c|c|c|c|c|c|c|c|c|c|c|c|c|}
\hline & Température & Conductivité & pH & Nitrite & Nitrate & Ammonium & Sulfate & Bicarbonate & Calcium & Magnésium & Sodium & Potassium \\
\hline Température & 1 & & & & & & & & & & & \\
\hline Conductivité & $-0,133$ & 1 & & & & & & & & & & \\
\hline $\mathrm{pH}$ & 0,030 & 0,280 & 1 & & & & & & & & & \\
\hline Nitrite & 0,386 & $-0,092$ & $-0,329$ & 1 & & & & & & & & \\
\hline Nitrate & $-0,171$ & 0,540 & $-0,504$ & 0,234 & 1 & & & & & & & \\
\hline Ammonium & 0,017 & 0,716 & $-0,182$ & 0,220 & 0,740 & 1 & & & & & & \\
\hline Sulfate & $-0,045$ & 0,433 & 0,465 & $-0,247$ & $-0,235$ & $-0,129$ & 1 & & & & & \\
\hline Bicarbonate & 0,154 & 0,689 & 0,298 & 0,126 & 0,209 & 0,628 & 0,242 & 1 & & & & \\
\hline Calcium & $-0,163$ & 0,664 & 0,592 & $-0,402$ & $-0,141$ & 0,010 & 0,856 & 0,371 & 1 & & & \\
\hline Magnesium & $-0,050$ & 0,591 & 0,583 & $-0,442$ & $-0,089$ & 0,109 & 0,310 & 0,404 & 0,702 & 1 & & \\
\hline Sodium & $-0,327$ & 0,803 & $-0,090$ & $-0,119$ & 0,710 & 0,770 & $-0,020$ & 0,448 & 0,319 & 0,468 & 1 & \\
\hline Potassium & $-0,152$ & 0,664 & $-0,319$ & $-0,074$ & 0,684 & 0,828 & $-0,101$ & 0,507 & 0,141 & 0,284 & 0,887 & 1 \\
\hline
\end{tabular}




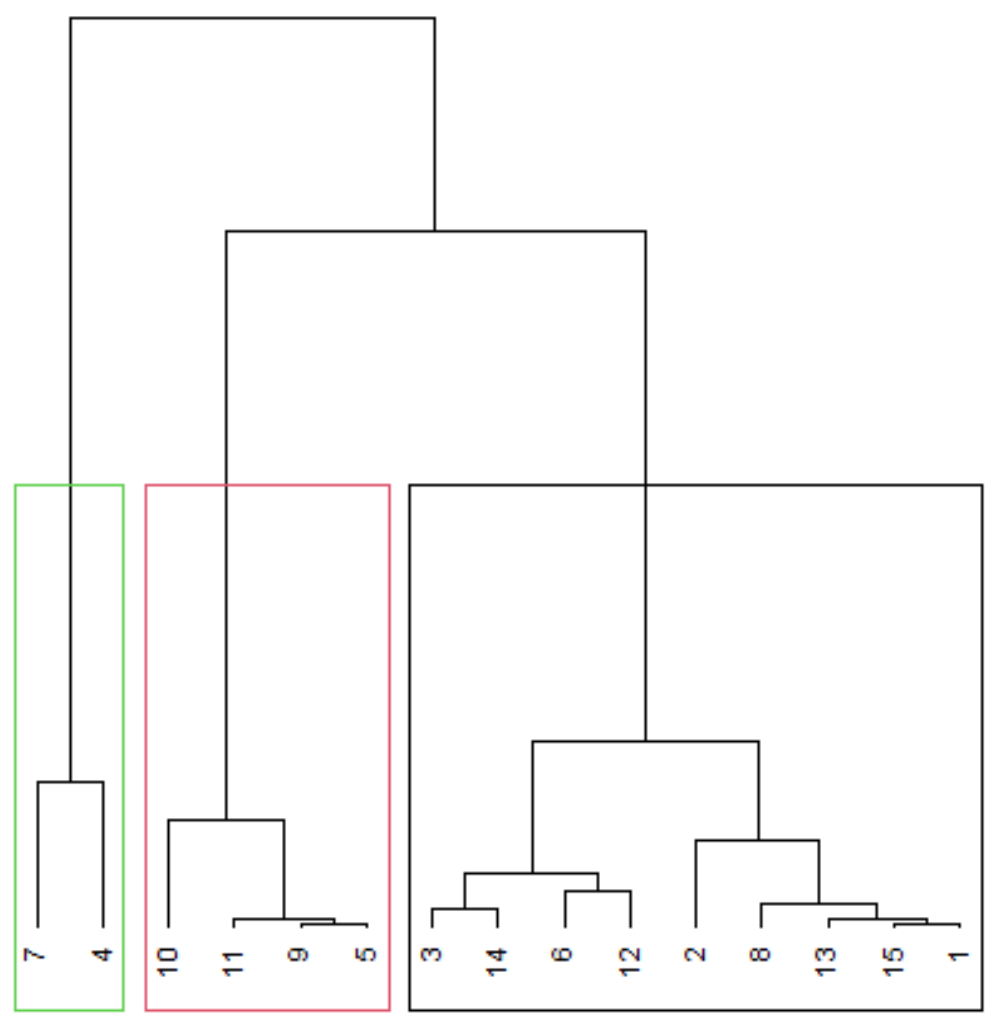

Figure 3 : Dendrogramme représentant la classification hiérarchique ascendante des 15 forages obtenue à partir des paramètres physico-chimiques de l'eau.

\section{DISCUSSION}

La présente étude se propose de caractériser la qualité physico-chimique des eaux de forages prisées par les populations de la ville de Daloa au détriment de l'eau fournie par la société de distribution d'eau de la ville. De façon générale, les paramètres mesurés sont conformes aux normes de l'OMS. La température moyenne des eaux de forage de la ville est de $30,0 \pm 1,6{ }^{\circ} \mathrm{C}$. Les valeurs de températures relevées sont en accord avec ce qui est rapporté dans la littérature. En effet, les eaux souterraines sont moins sensibles aux variations de température que les eaux superficielles (Rodier, 2009). D'une manière générale, les valeurs de $\mathrm{pH}$ obtenues pour ces eaux souterraines sont conformes aux résultats obtenus par d'autres études publiées dans la littérature car dans la plupart des eaux souterraines, le $\mathrm{pH}$ est compris entre 6 et 8,5
(Derwich et al., 2010; Belghiti et al., 2013; Mahamane et al., 2015). Les eaux des forages F1 $(5,97), \quad F 2 \quad(5,42)$ et F3 $(4,96)$ sont légèrement acides. Cette acidité peut leur conférer une propriété corrosive du ciment ou des métaux de canalisation. Ces eaux de Daloa sont faiblement minéralisées et convenables pour la consommation avec une moyenne de conductivité électrique de 246,2 $\pm 162,6$ $\mu \mathrm{S} / \mathrm{cm}$. Des valeurs de conductivité similaires sont obtenues par Mahamane et al. (2015) qui a étudié les caractéristiques des eaux souterraines de Yamtenga au Burkina Faso. Les teneurs en cations majeurs: $\mathrm{Ca}^{2+}, \mathrm{Mg}^{2+}$, $\mathrm{Na}^{+}$et $\mathrm{K}^{+}$sont en dessous des normes OMS pour ces valeurs. Ils sont sans danger pour la santé. Le calcium est extrêmement répandu dans la nature et en particulier dans les roches calcaires sous forme de carbonates. C'est le composant majeur de la dureté de l'eau. Le 
magnésium constitue le deuxième élément significatif de cette dureté. Nos échantillons d'eau présentent des teneurs en calcium et magnésium largement en dessous de la norme OMS. Les eaux de forage de la ville de Daloa sont ainsi des eaux douces ne présentant pas des inconvénients sur l'état des canalisations. Les anions majeurs $\mathrm{HCO}_{3}{ }^{-}, \mathrm{NO}_{3}{ }^{-}$et $\mathrm{SO}_{4}{ }^{2-}$ présentent également des teneurs en dessous des normes OMS. La concentration moyenne en bicarbonate est de 49,1 $\pm 20,8 \mathrm{mg} / \mathrm{L}$. Des concentrations élevées en bicarbonates donnent une saveur salée à l'eau (Mahamane et al., 2015). Les eaux des forages de Daloa présentent une concentration moyenne en nitrate de $41,6 \pm 38,89 \mathrm{mg} / \mathrm{L}$. Cette teneur indique que ces eaux sont de bonne qualité. Cependant, les forages 2, 4 et 7 présentent des concentrations supérieures à la norme. Ces teneurs seraient dues à la nitratation de $\mathrm{NH}_{4}{ }^{+}$ contenu dans les feldspaths des roches souterraines (Soro et al., 2010 ; Adjiri et al., 2019). Aussi, selon Yapo et al. (2010), les nappes profondes pourraient-elles s'enrichir en nitrate par appel d'eaux des nappes superficielles et d'eaux de surfaces contaminées par des polluants de surface.

\section{Conclusion}

$\mathrm{Au}$ terme de cette étude, les caractéristiques physico-chimiques des eaux des forages ont été déterminées dans la ville de Daloa. Ces eaux peuvent être classifiées selon les valeurs de $\mathrm{pH}$ et de conductivité. Les paramètres étudiés de ces eaux des forages sont conformes aux directives de l'OMS. Elles peuvent ainsi constituer une ressource alternative en eau de boisson pour les populations de la ville de Daloa.

\section{CONFLIT D'INTÉRÊTS}

Les auteurs ne déclarent aucun conflit d'intérêts en lien avec le présent article.

\section{CONTRIBUTIONS DES AUTEURS}

YFK est l'investigateur principal de ce manuscrit. NJAK a contribué aux prélèvements des échantillons. ABBK et SSK ont contribué aux analyses des résultats ainsi qu'à leur interprétation et discussion. ESA a participé à la lecture et la correction du manuscrit. OBY et TG ont assuré la supervision générale des travaux.

\section{REFERENCES}

Adjiri OA, Koné B, Aka N, Djabakaté I, Dibi B. 2019. Caractérisation physicochimique et source de la minéralisation des eaux souterraines des départements de Daloa et Zoukougbeu, Côte d'Ivoire. Int. J. Biol. Chem. Sci., 13(4): 2388-2401. DOI:

https://dx.doi.org/10.4314/ijbcs.v13i4.40

Aké DF, Coulibaly M, Niamke GM, Santos DS. 2018. La problématique de l'approvisionnement en eau potable et le développement des maladies a transmission hydrique dans les quartiers d'extension Orly de la ville de Daloa (Côte d'Ivoire). Revue Espace, Territoires, Sociétés et Santé, 1(2) : 91108.

CEAEQ (Centre d'Expertise en Analyse Environnementale du Québec). 2011. Détermination des métaux : méthode par spectrométrie de masse à source ionisante au plasma d'argon. Ministère de l'Environnement et de la Lutte contre les Changements Climatiques du Québec, p.32.

CICR (Comité International de la CroixRouge). 2012. Réalisation et réhabilitation de forages dans les conditions du terrain, Revue Technique, p.144.

Belghiti ML, Chahlaoui A, Bengoumi D. 2013. Caractéristiques physico-chimiques des eaux de certains puits utilisés comme source d'eau potable en milieu rural dans la région de Meknes (MAROC). Science Lib Editions Mersenne, 5: 1-16.

Belkhiri L, Boudoukha A, Mouni L, Baouz T. 2010. Multivariate statistical characterization of groundwater quality in Ain Azel plain, Algeria. African Journal of Environmental Science and Technology, $\quad 4(8): \quad 526-534$. DOI: 10.4314/ajest.v4i8.71307 
Derwich E, Benaabidate L, Zian A, Sadki O, Belghity D. 2010. Caractérisation physico-chimique des eaux de la nappe alluviale du Haut Sebou en aval de sa confluence avec OUED FES. Larhyss Journal, 8: 101-112.

Die K. 2006. Renforcement de l'alimentation en eau potable de la ville de Daloa à partir du barrage de Buyo en Côte d'Ivoire. Mémoire de fin de formation en DESS Génie Sanitaire et Environnement, Institut international de l'Ingénierie de l'Eau et de l'Environnement (2IE) de Ouagadougou, Burkina Faso, p.77.

Koné J, Yéo AP, Koné NY. 2018. L'eau est une préoccupation majeure en Côte d'Ivoire. Centre de Recherche et de Formation sur le Développement Intégré (CREFDI) à Abidjan, Afrobaromètre $\mathrm{N}^{\circ} 218$, p. 8.

Koukougnon WG. 2012. Milieu urbain et accès à l'eau potable cas de Daloa (CentreOuest de la Cote d'Ivoire). Thèse de Doctoral Unique, UFR Science de l'Homme et de la Société, Université Félix Houphouët Boigny, Abidjan, Côte d'Ivoire, p. 416.

Mahamane AA, Guel B. 2015.Caractérisations physico-chimiques des eaux souterraines de la localité de Yamtenga (Burkina Faso). Int. J. Biol. Chem. Sci., 9(1): 517533.

DOI:http://dx.doi.org/10.4314/ijbcs.v9i1. 44

OMS (Organisation Mondiale de la Santé). 2006. Water and Sanitation Related Diseases fact, troisième édition, Volume 1, Recommendations Geneva, World
Health Organization, Genève, Suisse, p.247.

OMS (Organisation Mondiale de la Santé). 2011. Directives de qualité pour l'eau de boisson. Quatrième édition. Publication Organisation Mondiale de la Santé. Genève, Suisse, 307-447.

ONU (Organisation des Nations Unies). 2019. Rapport sur les objectifs de développement durable.

RGPH (Recensement Général de la Population et de l'Habitat). 2014. Rapport d'exécution et présentation du résultat, Ministère du Plan et du Développement, Côte d'Ivoire, p.29.

Rodier J. 2009. L'Analyse de l'Eau (9 ${ }^{\text {ème }}$ édn). Collection Dunod, Technique Et Ingénierie: Paris.

Soro N, Ouattara L, Dongo K, Kouadio EK, Ahoussi K, Soro G, Oga MS, Savané I, Biemi J. 2010. Déchets municipaux dans le District d'Abidjan en Côte d'Ivoire: sources potentielles de pollution des eaux souterraines. Int. J. Biol. Chem. Sci., 4(6): 2203-2219.

DOI: 10.4314/ijbcs.v4i6.64952

Yapo OB, Mambo V, Seka A, Ohou MJA, Konan F, Gouzilé V, Tidou AS, Kouamé KV, Houenou P. 2010. Evaluation de la qualité des eaux de puits à usage domestique dans les quartiers défavorisés de quatre communes d'Abidjan (Côte d'Ivoire) : Koumassi, Marcory, PortBouet et Treichville. Int. J. Biol. Chem. Sci., $\quad$ 4(2): 289-307.DOI : 10.4314/ijbcs.v4i2.58111. 